\title{
Fine-pitch semiconductor detector for the FOXSI mission
}

\author{
S. Ishikawa, S. Saito, H. Tajima, T. Tanaka, S. Watanabe, H. Odaka, T. Fukuyama, M. Kokubun, T. Takahashi, Y. \\ Terada, S. Krucker, S. Christe, S. McBride and L. Glesener
}

\begin{abstract}
The Focusing Optics X-ray Solar Imager (FOXSI) is a NASA sounding rocket mission which will study particle acceleration and coronal heating on the Sun through high sensitivity observations in the hard X-ray energy band (5-15 keV). Combining high-resolution focusing $\mathrm{X}$-ray optics and fine-pitch imaging sensors, FOXSI will achieve superior sensitivity; two orders of magnitude better than that of the RHESSI satellite. As the focal plane detector, a Double-sided Si Strip Detector (DSSD) with a front-end ASIC (Application Specific Integrated Circuit) will fulfill the scientific requirements of spatial and energy resolution, low energy threshold and time resolution. We have designed and fabricated a DSSD with a thickness of $500 \mu \mathrm{m}$ and a dimension of $9.6 \mathrm{~mm} \times 9.6 \mathrm{~mm}$, containing 128 strips with a pitch of $75 \mu \mathrm{m}$, which corresponds to 8 arcsec at the focal length of $2 \mathrm{~m}$. We also developed a low-noise ASIC specified to FOXSI. The detector was successfully operated in the laboratory at a temperature of $-20^{\circ} \mathrm{C}$ and with an applied bias voltage of $300 \mathrm{~V}$, and the energy resolution of $430 \mathrm{eV}$ at a $14 \mathrm{keV}$ line was achieved. We also demonstrated fine-pitch imaging successfully by obtaining a shadow image, hence the implementation of scientific requirements was confirmed.
\end{abstract}

\section{INTRODUCTION}

$\mathbf{I}$ $\mathrm{N}$ solar flares, it is well known that electrons and ions are accelerated to high energies[1]. Accelerated particles emit hard X-rays (HXRs) by the bremsstrahlung process as they travel and lose their energy in the solar atmosphere. Therefore, HXR observations of the Sun provide important information about the energy release process in solar flares. Past solar HXR observations, such as those by the Hard Xray Telescope onboard the Yohkoh satellite [2] [3] and those by the Reuven Rematy High Spectroscopic Imager (RHESSI) satellite [4], have made use of non-focusing imaging techniques such as rotation modulation collimator imaging[5]. However, rotation modulation collimators require image reconstructions which limit dynamic range, and also require a large detector volume to obtain sufficient effective area, resulting in high background.

Manuscript received,-- 2010

S. Ishikawa, S. Saito, S. Watanabe, H. Odaka, T. Fukuyama, S. Sugimoto and T. Takahashi are with Institute of Space and Astronautical Science, Japan Aerospace Exploration Agency, Sagamihara, Kanagawa, Japan, and also with Department of Physics, University of Tokyo, Bunkyo, Tokyo, Japan (e-mail: ishikawa@astro.isas.jaxa.jp).

M. Kokubun is with Institute of Space and Astronautical Science, Japan Aerospace Exploration Agency, Sagamihara, Kanagawa, Japan.

Y. Terada is with Department of Physics, Saitama University, Japan.

H. Tajima and T. Tanaka are with Kavli Institute for Particle Astrophysics and Cosmology, Stanford University, Stanford, CA, USA.

S. Krucker, S. McBride and L. Glesener are with the Space Science Laboratory, U.C. Berkeley, CA, USA.

S. Christe is with NASA/Goddard Space Flight Center, MD, USA
Grazing-incidence HXR focusing optics are a promising new technology to avoid shortcomings of rotation modulation collimators. Focusing optics do not require image reconstructions and enable the use of small focal plane detectors while retaining large effective area, which imply a drastic reduction in non-solar background such as the one due to cosmicray, thereby increasing the sensitivity to solar HXR sources. A new sounding rocket mission, the Focusing Optics X-ray Solar Imager (FOXSI) will test out grazing-incidence HXR focusing optics [6] combined with position-sensitive focal plane detectors for solar observations (Fig. 1). FOXSI will achieve superior sensitivity, two orders of magnitude better than that of RHESSI around $10 \mathrm{keV}$. The FOXSI rocket is to be launched in October 2011.

To meet the science objectives of the FOXSI mission, a focal plane detector needs to satisfy the following requirements: A spatial resolution of $<116 \mu \mathrm{m}$ (corresponding to the angular resolution of the optics of $\sim 12$ arcseconds), an energy resolution of below $\sim 1 \mathrm{keV}$ (FWHM) and good photoabsorption efficiency (better than 50\%) in the $5-15 \mathrm{keV}$ band (see Table I). The focal plane detector also needs to be able to perform quasi-single photon counting for count rates up to $\sim 100$ counts/s expected from the quiet Sun. Existing imaging technologies widely used in focusing X-ray optics do not meet all of the above requirements. For example, chargecoupled devices can achieve good energy resolutions and low threshold energies, however, their time resolution is limited to an order of $1 \mathrm{~s}$ [7]. Therefore, it is critical to develop a new focal plane detector for FOXSI.

A Double-sided Silicon Strip Detector (DSSD) in combination with a low noise ASIC (Application Specific Integrated Circuit) is one of a few sensor technologies that could satisfy the requirements for the focal plane detector in FOXSI. We have developed a fine-pitch DSSD with a new detector structure to achieve the low noise requirement of this mission. In this paper, we present a detailed concept of the proposed solution, including experimental results to confirm its performance.

\section{Design of the FOXSI Focal Plane Detector}

\section{A. Double-sided Silicon Strip Detector}

In order to take advantage of the good angular resolution of 12 arcseconds of the FOXSI HXR focusing optics, we designed and fabricated a fine-pitch DSSD specific for FOXSI (the FOXSI DSSD). As the material for the semiconductor imaging detector, silicon has a sufficient photoabsorption 


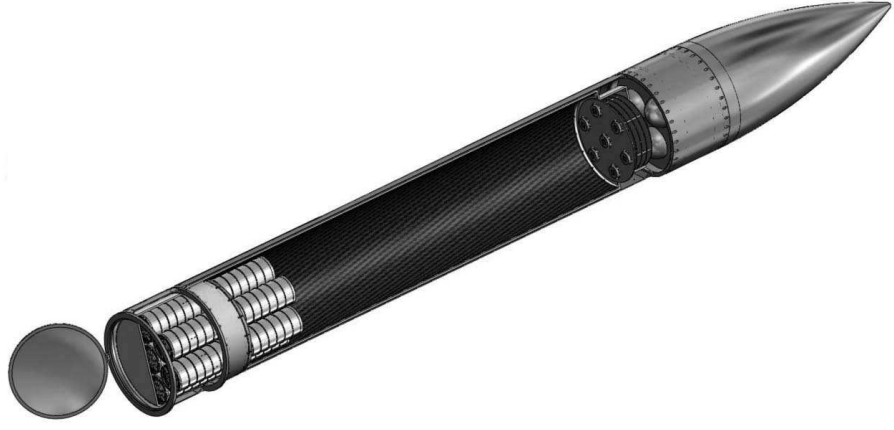

Fig. 1. Schematic of the FOXSI payload which consists of 7 telescope modules located at the optics plane (left) and 7 detectors at the the focal plane (right). Various electronics are located behind the focal plane. The focal length is $2 \mathrm{~m}$.

\begin{tabular}{ll}
\hline Energy range & $\sim 5$ to $15 \mathrm{keV}$ \\
Energy resolution (FWHM) & $\sim 1 \mathrm{keV}$ \\
Focal Length & $2 \mathrm{~m}$ \\
Angular resolution (FWHM) & 12 arcseconds \\
Field of view (HPD) & $640 \times 640$ arcseconds \\
Effective area & $120 \mathrm{~cm}^{2}(8 \mathrm{keV}) \sim 10 \mathrm{~cm}^{2}(15 \mathrm{keV})$ \\
Sensitivity & $0.004 \mathrm{~cm}^{-2} \mathrm{~s}^{-1} \mathrm{keV}^{-1}(\sim 8 \mathrm{keV})$ \\
Dynamic range & 100 for source separation $>30$ arcseconds \\
Observation time & $\sim 360 \mathrm{~s}$ \\
Launch site & White Sands Missile Range, NM, USA \\
Launch date & late 2011 \\
\hline & TABLE I \\
& FOXSI OVERVIEW [8].
\end{tabular}

efficiency in the energy band of interest. Although other materials such as cadmium telluride or cadmium zinc telluride have higher efficiencies and can achieve good performances at temperatures around $-20^{\circ} \mathrm{C}$, silicon is a well-researched material available in high purity, and detectors with high performances can be obtained with high yield at low cost. In addition, silicon detectors are quite radiation tolerant, and we have many years of experience in operating them in high radiation environments.

The FOXSI DSSD has highly acceptor doped $\left(\mathrm{p}^{+}\right)$silicon strips (p-side) and donor doped $\left(\mathrm{n}^{+}\right)$silicon strips (n-side) implanted orthogonally on a n-type silicon wafer (n-bulk). Each $\mathrm{n}$-side strip is surrounded by a floating $\mathrm{p}^{+}$-doped implantation (p-stop) to insulate it from adjacent strips. Aluminum electrodes are directly coupled to each strip with an ohmic contact. DSSDs have been widely developed for astrophysical and nuclear physics applications [9][10]. A DSSD with a strip pitch of $250 \mu \mathrm{m}$ or $400 \mu \mathrm{m}$ has been developed by our group as a scattering detector of a Si/CdTe Compton camera[10], [11], [12]. In this development, we added aluminum electrodes DCcoupled to p-stops in order to minimize the p-stop resistance since it generates Johnson noise. A similar DSSD is also being developed for the Hard X-ray Imager on board the Japanese $\mathrm{X}$-ray astronomy satellite $A S T R O-H[13]$, [14].

The active area of the FOXSI DSSD is $9.6 \mathrm{~mm} \times 9.6 \mathrm{~mm}$ and the number of strips is 128 for $\mathrm{p}$-side and $\mathrm{n}$-side, providing position information for $128 \times 128=16384$ pixels by reading out only $128+128=256$ channels, resulting in lower power consumption. The pitch of the strips is $75 \mu \mathrm{m}$, corresponding

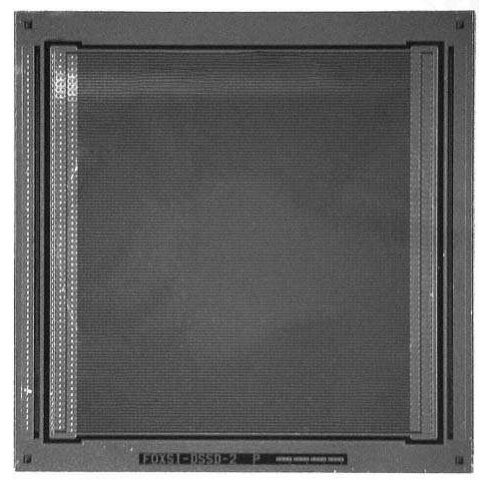

Fig. 2. Photograph of the $128 \times 128$ FOXSI DSSD (p-side is shown). The active area is $9.6 \mathrm{~mm} \times 9.6 \mathrm{~mm}$, and the strip pitch is $75 \mu \mathrm{m}$.

\begin{tabular}{ll}
\hline Active Area & $9.6 \mathrm{~mm} \times 9.6 \mathrm{~mm}$ \\
Thickness & $500 \mu \mathrm{m}$ \\
Strip pitch & $75 \mu \mathrm{m}$ \\
Number of strips per side & 128 \\
\hline
\end{tabular}

TABLE II

SPECIFICATIONS OF THE FOXSI DSSD.

to an angular resolution of 8 arcseconds at the focal length of $2 \mathrm{~m}$. Hence, the spatial resolution of the optics is oversampled by a factor of 1.5. The thickness is $500 \mu \mathrm{m}$, which implies a photoabsorption efficiency of $98 \%$ for $10 \mathrm{keV}$ and $68 \%$ for $15 \mathrm{keV}$. Guard-ring electrodes with a width of $100 \mu \mathrm{m}$ are implemented on both sides to block the leakage current from the periphery. Fig. 2 shows a photo, and Table II shows the specifications of the FOXSI DSSD. The device is manufactured by Hamamatsu Photonics, Japan.

\section{B. The Low Noise Front End ASIC VATA45I}

To achieve good energy resolutions and a low threshold energy, a 64-channel analog ASIC, VATA451, has been developed by ISAS, KIPAC and GM-IDEAS based on a low noise front end ASIC previously developed for Compton cameras for applications including ASTRO-H[15]. Fig. 3 shows a function block diagram for VATA451. Each channel of the analog circuit of the VATA451 consists of a charge-sensitive amplifier (CSA) followed by two shaping amplifiers, one with a fast shaping time for generating triggers (TA section), and the other with a slow shaping time for charge measurements (VA section). If the output signal from the fast shaper of one or more channels is greater than a threshold value in the TA section, the output signals from the slow shaper of each channel will be sampled and held with an appropriate delay. A Wilkinson-type analog-to-digital converter, by which all channels can be digitized in parallel, is also included in the VATA451. The ADC is 10 bit and takes $100 \mu$ s for digitization with a $10 \mathrm{MHz}$ clock, and output signals are multiplexed. A common-mode noise calculator is also built into the ASIC. Common-mode noise, which is a noise component common to all channels of an ASIC for a given event, can be obtained by using the median value of the signals from all channels for that event. These analog and ADC architectures are functionally the same as the former ASIC, VA32TA6[16]. 
This ASIC is specifically optimized for the FOXSI mission as follows. The gain of the CSA is higher than that of ASTRO$H$ to achieve better noise performance since the energy range of FOXSI (below $15 \mathrm{keV}$ ) is lower than that of ASTRO-H. The fast shaper provides a longer shaping time constant option for generating triggers, which will be beneficial to achieve a low threshold energy required by FOXSI. The time constant of the slow shaper can be adjusted from $2 \mu$ s to $4 \mu$ s, and that of the fast shaper can be set to $0.6 \mu \mathrm{s}$ or $1.2 \mu \mathrm{s}$.

The input FET of the VATA451 is optimized to minimize the noise for an input capacitance of $5 \mathrm{pF}$ and a leakage current of $10 \mathrm{pA}$ within a power constraint of $1 \mathrm{~mW} /$ channel, resulting in an equivalent noise charge (ENC) of $64 e^{-}$(RMS) for such input loads with a shaping time of $3 \mu \mathrm{s}$.

Since there are 128 strips for each p-side and n-side, two ASICs for each side are required, or four ASICs are needed to read out one FOXSI DSSD. To connect from the DSSD to the ASICs, each DSSD strip is wire-bonded to the readout pads of the ASICs.

\section{BASIC PROPERTIES OF A DSSD}

We measured the leakage current of one central p-side strip of the FOXSI DSSD at temperatures of $15^{\circ} \mathrm{C}, 0^{\circ} \mathrm{C}$ and $-20^{\circ} \mathrm{C}$ (Nominal in-flight temperature is $-20^{\circ} \mathrm{C}$ ). Fig. 4 shows the measured $I-V$ characteristic. The leakage current is found to be proportional to $\exp (-1 / k T)$. This is consistent with the idea that the origin of the current is thermal effect. Under $-20^{\circ} \mathrm{C}$, the leakage current is measured to be $\sim 1.5 \mathrm{pA}$ with bias voltages of $>200 \mathrm{~V}$. The corresponding ENC is $\sim 7 e^{-}$ (RMS) at a shaping time of $3 \mu$ s calculated by a formula $\sim 110 \sqrt{I \tau} e^{-}$where $I$ is the leakage current in $\mathrm{nA}$ and $\tau$ is the time constant of a readout $C R-R C$ circuit in $\mu \mathrm{s}$. This result implies that the temperature of $-20^{\circ} \mathrm{C}$ is sufficiently low to obtain the best performance because the noise from the leakage current is negligible compared to normal readout noise.

Fig. 5 shows an C-V characteristic of the FOXSI DSSD at a room temperature. The body capacitance and the inter-strip capacitances of $\mathrm{p}$-side and $\mathrm{n}$-side were measured. An inter-strip capacitance is a capacitance between one strip and the surrounding strips. The data of inter-strip capacitance are plotted for central p-side and n-side strips. The amplifier noise due to the detector capacitance for a single strip can be parametrized as $\sqrt{20^{2}+\left(10.5+7 \times C_{\mathrm{d}}\right)^{2}+\left(12.2+8.1 \times C_{\mathrm{d}}\right)^{2} / \tau} e^{-}$, where $C_{\mathrm{d}}$ is the sum of the body capacitance per single strip and the inter-strip capacitance.

The body capacitance becomes almost constant with bias voltages above $\sim 100 \mathrm{~V}$, which implies that the DSSD is fully depleted at about $100 \mathrm{~V}$. The inter-strip capacitance of the $\mathrm{n}$-side can be measured above $80 \mathrm{~V}$, and becomes almost constant above $\sim 200 \mathrm{~V}$. This implies that $\mathrm{n}$-side strips will be isolated above $80 \mathrm{~V}$, and completely isolated above $\sim 200 \mathrm{~V}$. The $\mathrm{C}-\mathrm{V}$ characteristic suggests that a higher bias voltage such as $300 \mathrm{~V}$ is desired to reduce capacitance noise. The body capacitance for all strips is measured to be $22.4 \mathrm{pF}$, that is $0.2 \mathrm{pF}$ per single strip with a bias voltage of $300 \mathrm{~V}$. The interstrip capacitances of the $\mathrm{p}$-side and $\mathrm{n}$-side are measured to be 2.3 and $4.2 \mathrm{pF}$ at a bias voltage of $300 \mathrm{~V}$.

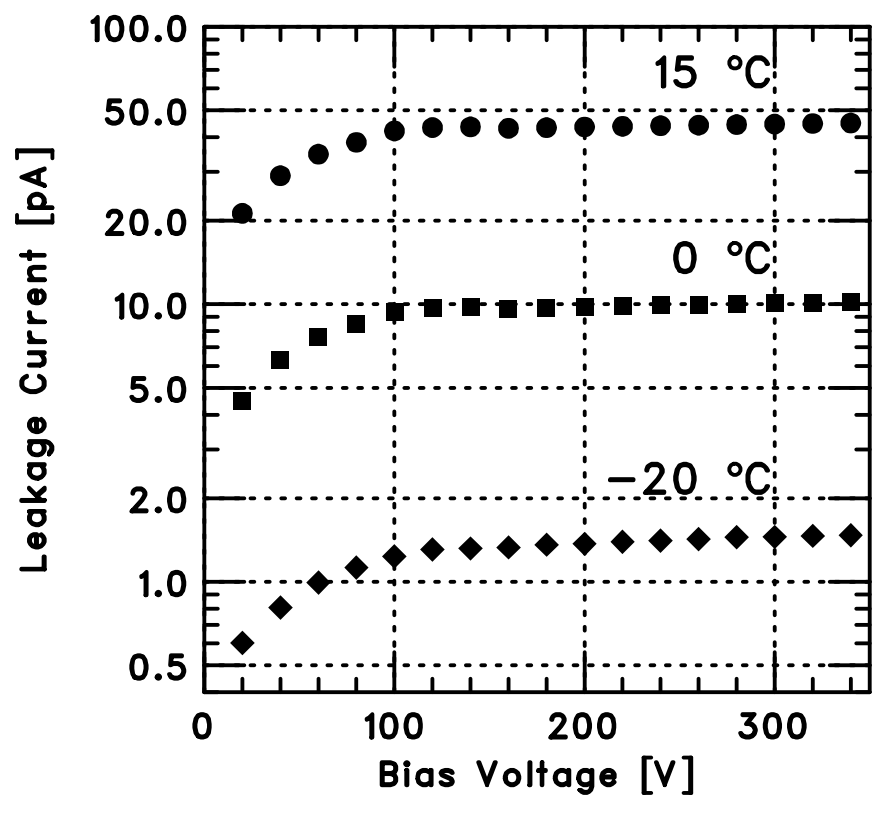

Fig. 4. I-V characteristic of FOXSI DSSD.

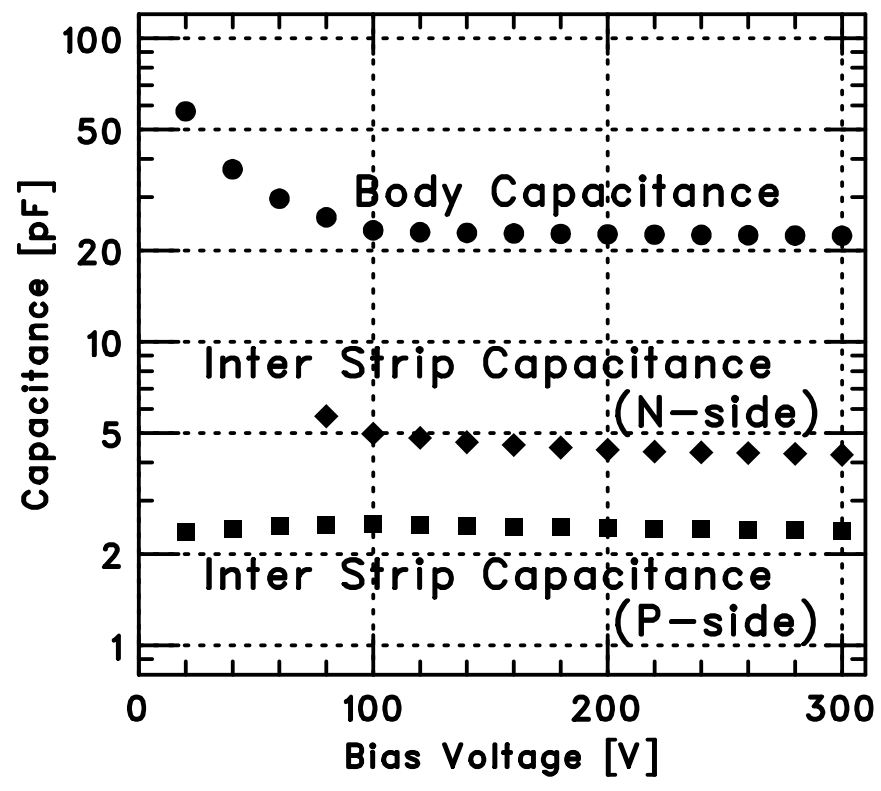

Fig. 5. C-V characteristic of FOXSI DSSD.

\section{Performance of a Prototype For the FOXSI Focal Plane Detector}

We have fabricated a prototype of the FOXSI focal plane detector that consists of one FOXSI DSSD and four VA451 ASICs. Fig. 6 shows a photo of the experimental setup. Wirebonding from the detector to the ASICs was performed by a semi-automatic wire-bonding machine in our laboratory. Due to operational errors, some wires failed to be bonded. Therefore, some strips are not connected to the ASICs and cannot be read out. Such strips are electrically floated.

Under a temperature of $-20^{\circ} \mathrm{C}$ and a bias voltage of $300 \mathrm{~V}$, we successfully operated the FOXSI DSSD. Events from 124 out of 128 strips of the p-side and 120 out of 128 strips for 


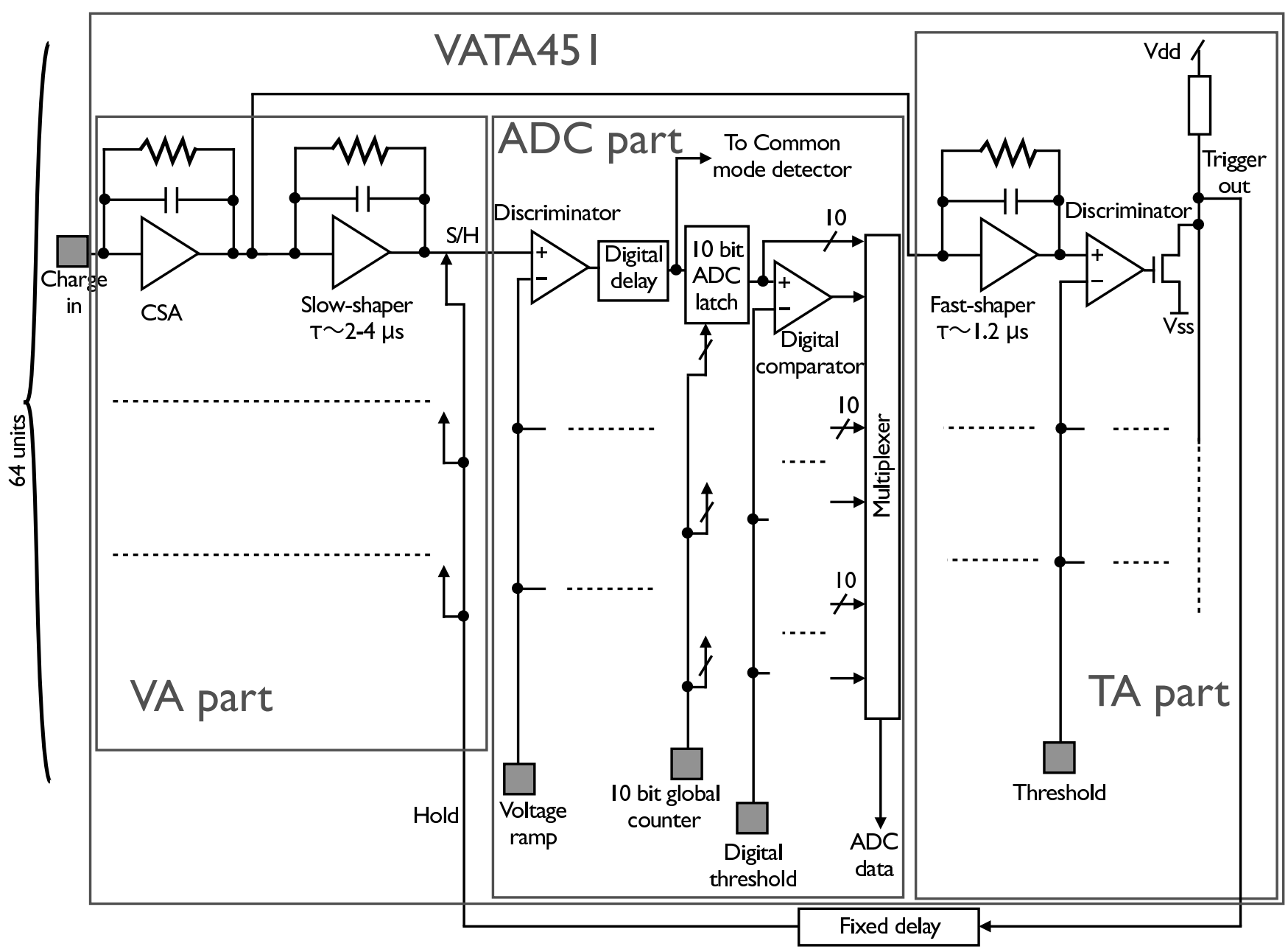

Fig. 3. Functional block diagram of the readout ASIC, VATA451. The ASIC consists of a pulse-shaping VA part and a trigger-generating TA parts. An ADC is also included.

the n-side were used for the following analysis. In the spectral analysis, we use only events where a single hit is detected above the threshold of $5 \mathrm{keV}$ in both the p-side and $\mathrm{n}$-side in order to suppress events with more than one interaction or any charge sharing between two adjacent strips.

Spectra from the $\mathrm{p}$-side and $\mathrm{n}$-side were obtained with the use of a ${ }^{241} \mathrm{Am}$ radioactive isotope source. The spectra of all active strips on the p-side (black curve) and n-side (gray curve) are shown in Fig. 7 The energy resolutions of the pside and n-side were measured to be $430 \mathrm{eV}$ and $1.6 \mathrm{keV}$ (FWHM) at $14 \mathrm{keV}$, respectively. Since we can use the better energy information from the p-side for the spectral analysis, it is confirmed that the energy resolution fulfills the mission requirement.

The distribution of energy resolutions (FWHM) of the p-side and n-side strips are shown in Fig. 8 The energy resolutions of most channels are distributed from 0.4 to $0.5 \mathrm{keV}$ on the p-side, excluding an exception with an energy resolution of $0.9 \mathrm{keV}$. The energy resolutions for the $\mathrm{n}$-side are distributed between 0.9 and $1.9 \mathrm{keV}$ with the exception of an outlier at $3.1 \mathrm{keV}$. Both outliers of the p-side and $\mathrm{n}$-side correspond to edge strips, which neighbor the guard ring. Since the energy resolution and its dispersion are much worse on the $\mathrm{n}$-side than on the $\mathrm{p}$-side, the strip structure on the $\mathrm{n}$-side is considered to be responsible for the excess noise on the n-side. In fact, addition of the DC-coupled aluminum electrode on the pstop improved the energy resolution. A remaining noise source could be an accumulation layer between p-stops which may act as a floating n-strip with high resistance. Fig. 9 shows the position dependence of the energy resolution where we observe a moderate bump structure peaking on the $\mathrm{n}$-side around strip numbers 70-90. Since the resistance of the accumulation layer is highly dependent on the fabrication process, it can reasonably explain the asymmetric bump observed here. On the other hand, we do not observe such structure or large dispersion in the energy resolution on $\mathrm{n}$-side with another DSSD sample for the HXI fabricated in the same batch. These observations may indicate that the excess noise on the $\mathrm{n}$-side originates from the accumulation layer and may depend on the location in the silicon wafer and fabrication process.

Although the energy resolution observed for the $14 \mathrm{keV}$ line on the $\mathrm{p}$-side is much better than that on the $\mathrm{n}$-side, it is still worse than the energy resolution expected from the noise performance of the ASIC, especially for higher 


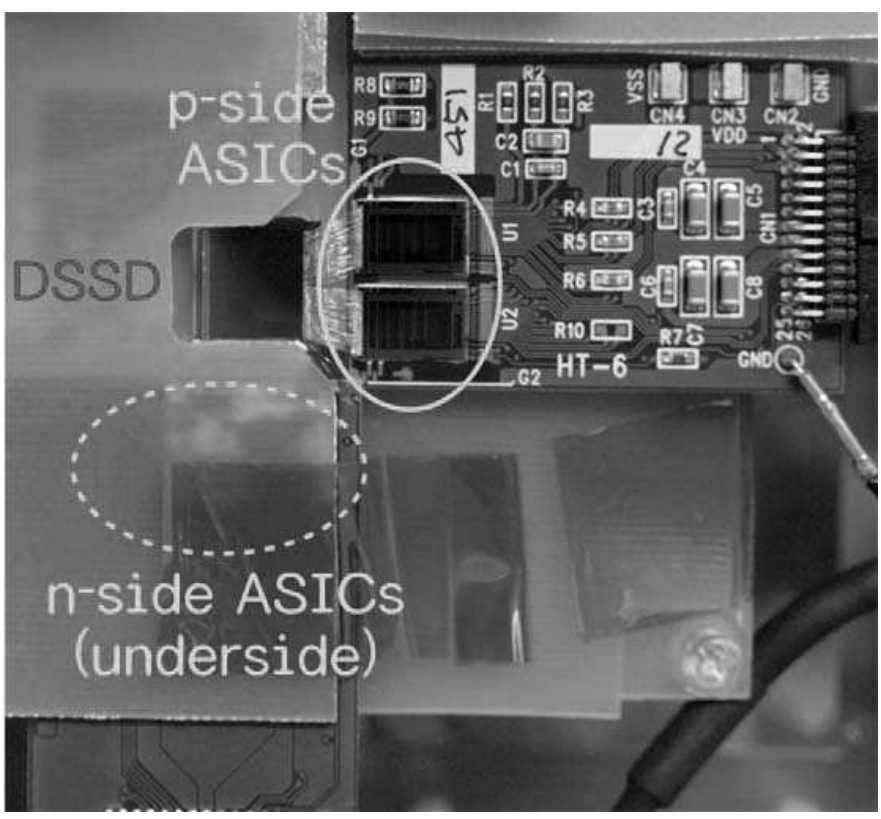

Fig. 6. Photo of the DSSD experimental setup. Each strip of the DSSD is connected to an ASIC by wire-bonding.

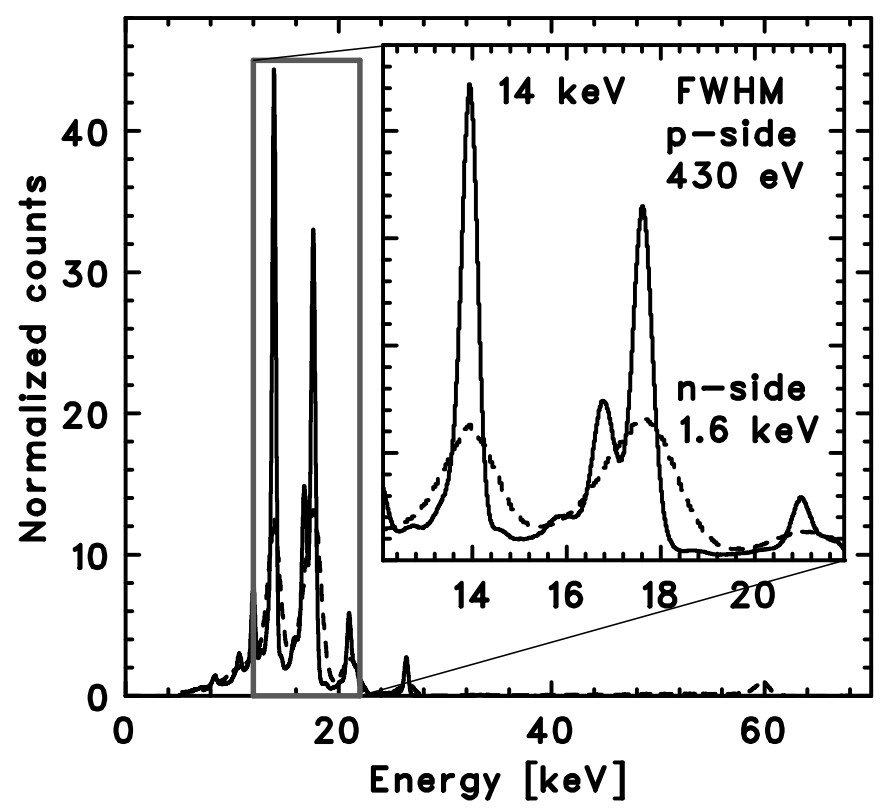

Fig. 7. Spectra from the p-side (solid line) and n-side (dashed line) of the FOXSI DSSD with a ${ }^{241} \mathrm{Am}$ source. The operating temperature was $-20^{\circ} \mathrm{C}$ and the bias voltage was $300 \mathrm{~V}$.

energies. In order to study the nature of the excess noise on the p-side, we study the energy dependence of the energy resolution as shown in Fig. 10, Data points are measured energy resolutions while a gray region gives the resolution calculated from the ASIC noise performance for a load with capacitance of $(3.0 \pm 0.5) \mathrm{pF}$ at a peaking time of $4.8 \mathrm{~s}$ $(346 \pm 28 \mathrm{eV})$ and Fano noise (fluctuation of electron hole pairs produced by ionization) where the load capacitasnce includes a parasitic capacitancer of $(0.5 \pm 0.5) \mathrm{pF}$. A fit to an empirical formula, $\sqrt{E_{0}^{2}+\left(f_{1} E\right)^{2}+N_{\text {Fano }}(E)^{2}}$ yields $E_{0}=365 \pm 15 \mathrm{eV}, f_{1}=(4.0 \pm 0.5) \times 10^{-3}$ where $N_{\text {Fano }}(E)$ is

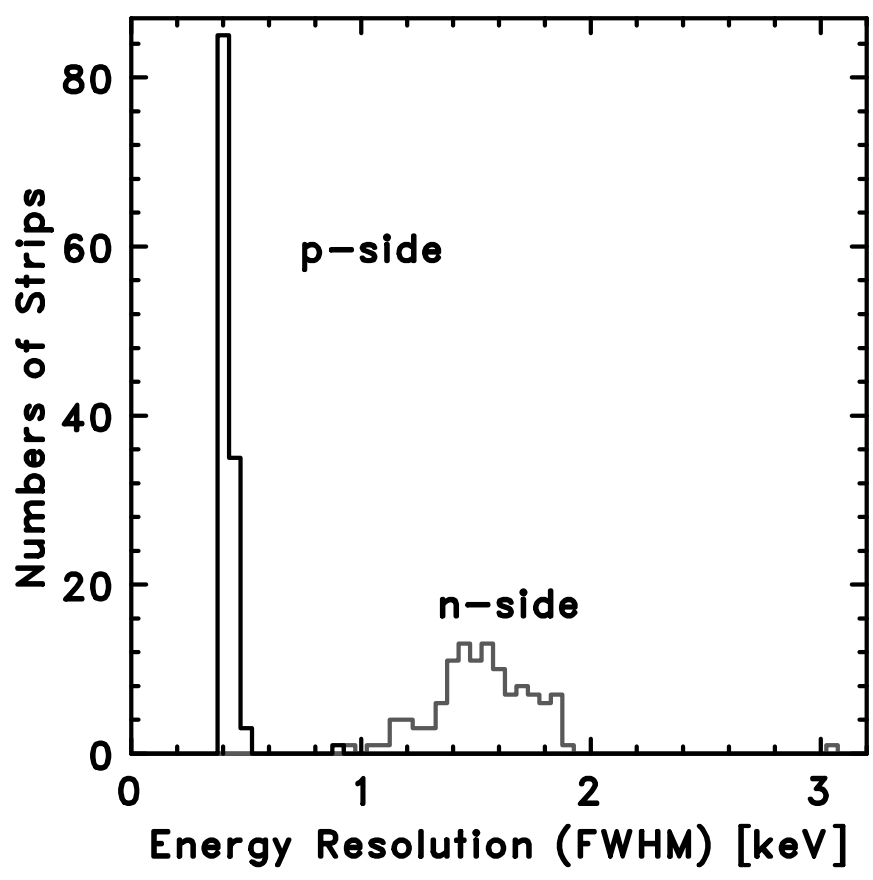

Fig. 8. Distribution of energy resolution (FWHM) for the $14 \mathrm{keV}$ line.

the Fano noise. The linear term may be explained by the gain uncertainties of the ASIC as mentioned in previous papers[10], [15].

While we can achieve a spectral resolution better than $<0.5 \mathrm{keV}$ in the FOXSI energy range solely due to a good noise performance on the $\mathrm{p}$-side, the localization of the incident X-ray position still relies on detecting X-ray signals on both the $\mathrm{p}$-side and the $\mathrm{n}$-side. Since the noise performance on the $\mathrm{n}$-side is marginal, we calculate the probability for wrong localizations. Assuming a Gaussian noise distribution, the energy resolution of $2.0 \mathrm{keV}$ for the n-side corresponds to a chance probability of $<10^{-7}$ for recording an energy above $5 \mathrm{keV}$ due to noise alone. This implies that positions of incident photons can be determined with a negligible probability of spurious hits. Therefore, we confirm that the DSSD can be operated as an imager at a low energy threshold of $5 \mathrm{keV}$, which fulfills the scientific requirements of the FOXSI mission.

Fig. 11 shows a shadow image of a tungsten plate with $1 \mathrm{~mm}$ pitch slits using X-rays from a ${ }^{133} \mathrm{Ba}$ source in an energy band from 20 to $40 \mathrm{keV}$. To test the imaging performance, the tungsten plate is tilted by $45^{\circ}$ and does not cover the top left part of the detector. The width of the slits is $100 \mu \mathrm{m}$. The individual slits are clearly visible, successfully demonstrating the capabilities of fine-pitch imaging and spectroscopy.

In the left part of the top edge of the image $(x \leq 90$ and $y \geq 120$ ), a longitudinal stripe pattern appears even though counts should be almost flat in this region since there is no mask. In the edges of the p-side strips (the top edge and the bottom edge of the image), wire-bonding pads are placed, which is considered to be the cause of the pattern. The bonding pads are wider than the strips, and therefore staggered. Events in this region can be used for spectroscopy although the spatial 

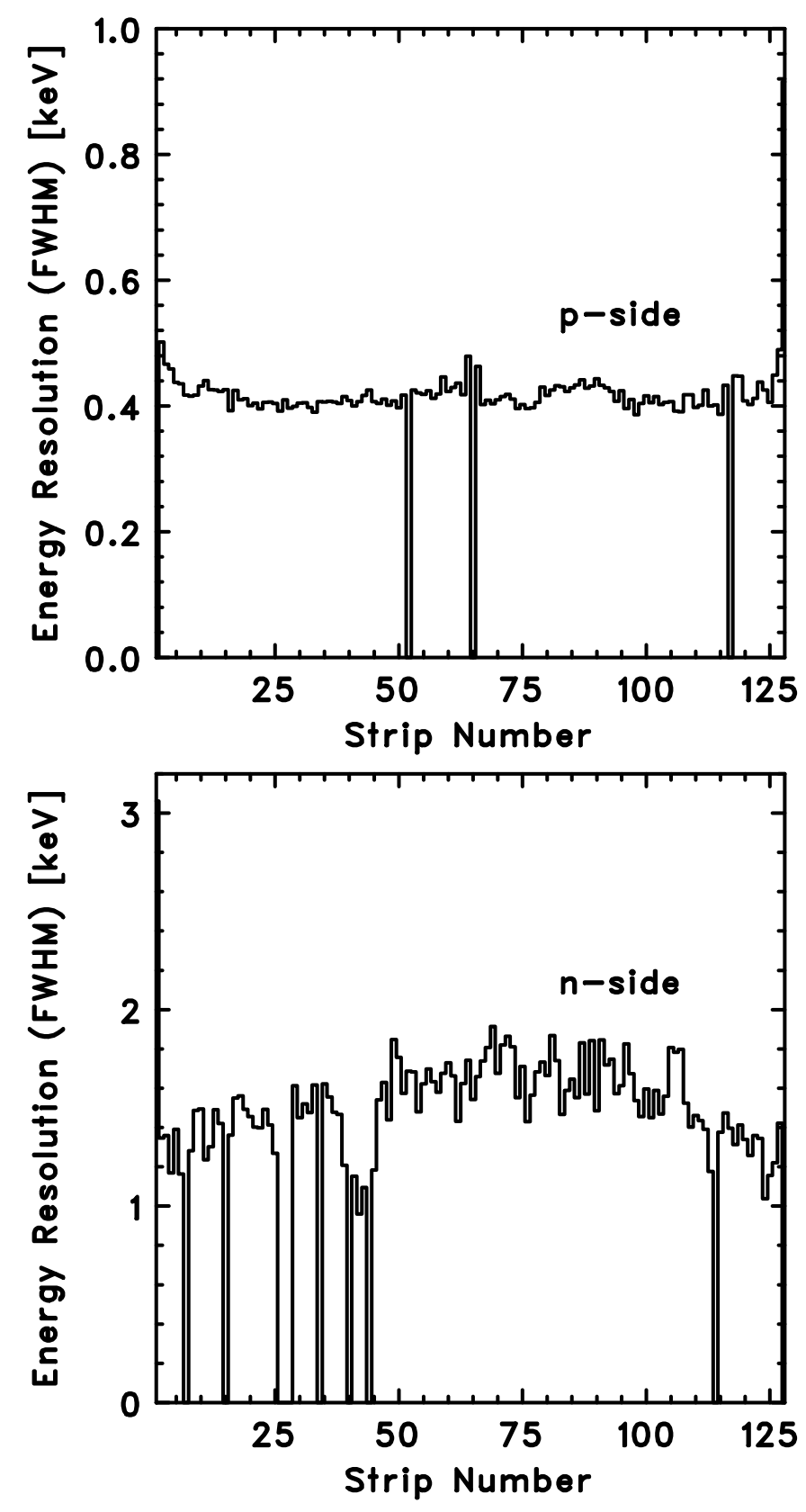

Fig. 9. Energy resolution (FWHM) for the $14 \mathrm{keV}$ line as a function of the strip number. Top: p-side. Bottom: n-side.

resolution for the $x$-position is worse than the other regions.

On the other hand, although signals are successfully obtained by the $\mathrm{p}$-side, the right and left edges on the image $(x \leq 2$ and $x \geq 125$ ) have no $\mathrm{n}$-side signal. This is considered to be caused by the presence of wire-bonding pads on $n$-side. In this region, events can be used only for spectroscopy.

\section{CONCLUSIONS}

FOXSI is a sounding rocket mission which will observe the Sun in the hard X-rays (5-15 keV) by using HXR focusing optics and a semiconductor imaging detector. We have developed fine-pitch and low noise DSSDs for the FOXSI focal plane detector, specifically to fulfill scientific requirements

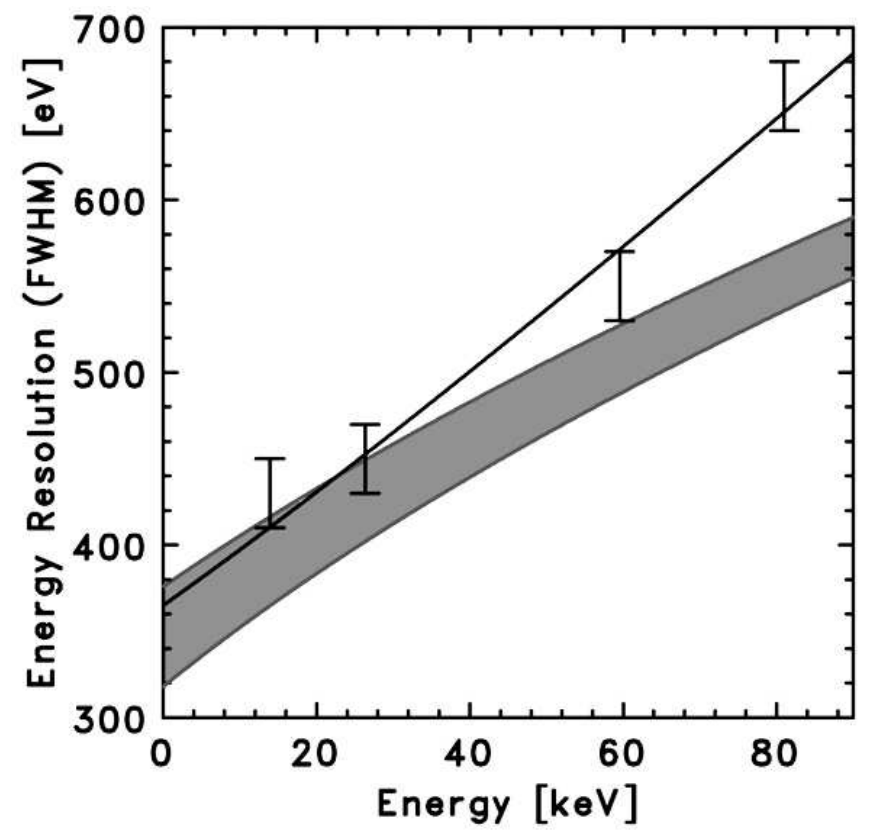

Fig. 10. Energy resolution (FWHM) as a function of the incident energy. Gray region indicate the energy resolution expected from the ASIC performance. Solid curve shows the fit result.

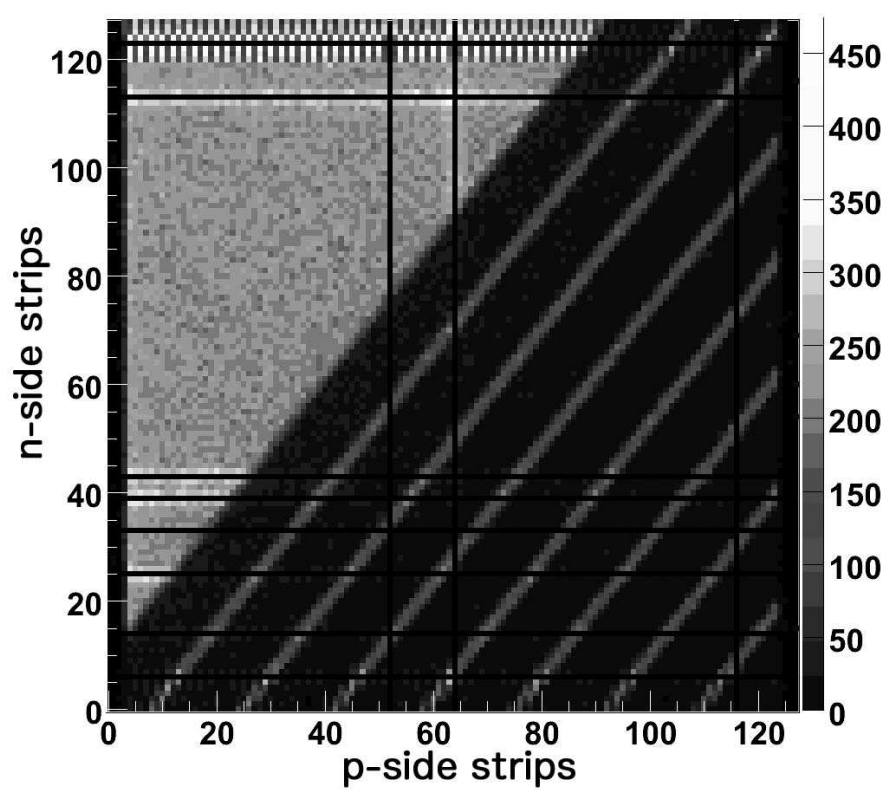

Fig. 11. Shadow image from the FOXSI DSSD with a radio isotope of ${ }^{133} \mathrm{Ba}$. The scale gives the number of counts. The width of the slits in this image is $100 \mu \mathrm{m}$.

on the spatial resolution, energy resolution, low threshold energy and time resolution. We designed and fabricated a DSSD with a thickness of $500 \mu \mathrm{m}$, and a dimension of $9.6 \times 9.6 \mathrm{~mm}$ containing 128 strips on a pitch of $75 \mu \mathrm{m}$, which corresponds to 8 arcsec at the focal length of $2 \mathrm{~m}$. The DSSD was successfully operated in a laboratory experiment under a temperature of $-20^{\circ} \mathrm{C}$ and a bias voltage of $300 \mathrm{~V}$. The energy resolution was measured to be $430 \mathrm{eV}$ for the p-side and $1.6 \mathrm{keV}$ for the n-side at $14 \mathrm{keV}$, sufficient for the FOXSI mission requirement. We also successfully obtained a shadow 
image and thus demonstrated the capabilities of both fine-pitch imaging and spectroscopy.

\section{REFERENCES}

[1] A. O. Benz, "Flare Observations," Living Reviews in Solar Physics, vol. 5, pp. 1-+, 2008.

[2] T. Kosugi, S. Masuda, K. Makishima, M. Inda, T. Murakami, T. Dotani, Y. Ogawara, T. Sakao, K. Kai, and H. Nakajima, "The hard X-ray telescope (HXT) for the Solar-A mission," Sol. Phys., vol. 136, pp. 1736, 1991.

[3] T. Kosugi, T. Sakao, S. Masuda, K. Makishima, M. Inda, T. Murakami, Y. Ogawara, K. Yaji, and K. Matsushita, "The Hard X-ray Telescope (HXT) onboard YOHKOH - Its performance and some initial results," PASJ, vol. 44, pp. L45-L49, 1992.

[4] R. P. Lin, B. R. Dennis, G. J. Hurford, D. M. Smith, A. Zehnder, P. R. Harvey, D. W. Curtis, D. Pankow, P. Turin, M. Bester, A. Csillaghy, M. Lewis, N. Madden, H. F. van Beek, M. Appleby, T. Raudorf, J. McTiernan, R. Ramaty, E. Schmahl, R. Schwartz, S. Krucker, R. Abiad, T. Quinn, P. Berg, M. Hashii, R. Sterling, R. Jackson, R. Pratt, R. D. Campbell, D. Malone, D. Landis, C. P. Barrington-Leigh, S. SlassiSennou, C. Cork, D. Clark, D. Amato, L. Orwig, R. Boyle, I. S. Banks, K. Shirey, A. K. Tolbert, D. Zarro, F. Snow, K. Thomsen, R. Henneck, A. McHedlishvili, P. Ming, M. Fivian, J. Jordan, R. Wanner, J. Crubb, J. Preble, M. Matranga, A. Benz, H. Hudson, R. C. Canfield, G. D Holman, C. Crannell, T. Kosugi, A. G. Emslie, N. Vilmer, J. C. Brown, C. Johns-Krull, M. Aschwanden, T. Metcalf, and A. Conway, "The Reuven Ramaty High-Energy Solar Spectroscopic Imager (RHESSI)," Sol. Phys., vol. 210, pp. 3-32, 2002.

[5] G. J. Hurford, E. J. Schmahl, R. A. Schwartz, A. J. Conway, M. J. Aschwanden, A. Csillaghy, B. R. Dennis, C. Johns-Krull, S. Krucker, R. P. Lin, J. McTiernan, T. R. Metcalf, J. Sato, and D. M. Smith, "The RHESSI Imaging Concept," Sol. Phys., vol. 210, pp. 61-86, 2002.

[6] B. D. Ramsey, C. D. A. J. A. Apple, C. M. Benson, K. L. Dietz, R. F. Elsner, D. E. Engelhaupt, K. K. Ghosh, J. J. Kolodziejczak, S. L. O’Dell, C. O. Speegle, D. A. Swartz, and M. C. Weisskopf, "First Images from HERO, a Hard X-Ray Focusing Telescope," ApJ, vol. 568, pp. 432-435, 2002.

[7] K. P. Singh, "Techniques in X-ray astronomy 2. Imaging detectors," Resonance, vol. 10, pp. 15-23, 2005.

[8] S. Krucker, S. Christe, L. Glesener, S. McBride, P. Turin, D. Glaser, P. Saint-Hilaire, G. Delory, R. P. Lin, M. Gubarev, B. Ramsey, Y. Terada, S. Ishikawa, M. Kokubun, S. Saito, T. Takahashi, S. Watanabe, K. Nakazawa, H. Tajima, S. Masuda, T. Minoshima, and M. Shimojo, "The Focusing Optics X-ray Solar Imager (FOXSI)," in Optics for EUV, $X$-Ray, and Gamma-Ray Astronomy IV, ser. Proc. SPIE, S. L. O'Dell and G. Pareschi, Eds., vol. 7437, 2009, p. 743705.

[9] P. J. Sellin, P. J. Woods, D. Branford, T. Davinson, N. J. Davis, D. G. Ireland, K. Livingston, R. D. Page, A. C. Shotter, S. Hofmann, R. A. Hunt, A. N. James, M. A. C. Hotchkis, M. A. Freer, and S. L. Thomas, "A double-sided silicon strip detector system for proton radioactivity studies," Nucl. Inst. Meth., vol. A 311, pp. 217-223, 1992.

[10] H. Tajima, T. Kamae, S. Uno, T. Nakamoto, Y. Fukazawa, T. Mitani, T. Takahashi, K. Nakazawa, Y. Okada, and M. Nomachi, "Low Noise Double-Sided Silicon Strip Detector for Multiple-Compton Gamma-ray Telescope," in X-Ray and Gamma-Ray Telescopes and Instruments for Astronomy, ser. Proc. SPIE, J. E. Truemper and H. D. Tananbaum, Eds., vol. 4851, 2003, pp. 875-884.

[11] S. Takeda, S. Watanabe, T. Tanaka, K. Nakazawa, T. Takahashi, Y. Fukazawa, H. Yasuda, H. Tajima, Y. Kuroda, M. Onishi, and K. Genba, "Development of double-sided silicon strip detectors (DSSD) for a Compton telescope," Nucl. Inst. Meth., vol. A 579, pp. 859-865, 2007.

[12] S. Watanabe, S. Tanekda, S. Ishikawa, H. Odaka, M. Ushio, T. Tanaka, K. Nakazawa, T. Takahashi, H. Tajima, Y. Fukazawa, Y. Kuroda, and M. Onishi, "Development of Semiconductor Imaging Detectors for a Si/CdTe Compton Camera," Nucl. Inst. Meth., vol. A 579, pp. 871-877, 2007.

[13] T. Takahashi, K. Mitsuda, R. Kelley, F. Aharonian, F. Akimotod, S. Allen, N. Anabuki, L. Angelini, K. Arnaud, H. Awaki, A. Bamba, N. Bando, M. Bautz, R. Blandford, K. Boyc, G. Brown, M. Chernyakova, P. Coppi, E. Costantini, J. Cottam, J. Crow, J. de Plaa, C. de Vries, J. den Herder, M. DiPirro, C. Done, T. Dotani, K. Ebisawa, T. Enoto, Y. Ezoe, A. Fabian, R. Fujimoto, Y. Fukazawa, S. Funk, A. Furuzawa, M. Galeazzi, P. Gandhi, K. Gendreau, K. Gilmore, Y. Haba, K. Hamaguchi, I. Hatsukade, K. Hayashida, J. Hiraga, K. Hirose,
A. Hornschemeier, J. Hughes, U. Hwang, R. Iizuka, K. Ishibashi, M. Ishida, K. Ishimura, Y. Ishisaki, N. Isobe, M. Ito, N. Iwata, J. Kaastra, T. Kallman, T. Kamae, H. Katagiri, J. Kataoka, S. Katsuda, M. Kawaharada, N. Kawai, S. Kawasaki, D. Khangaluyan, C. Kilbourne, K. Kinugasa, S. Kitamoto, T. Kitayama, T. Kohmura, M. Kokubun, T. Kosaka, T. Kotani, K. Koyama, A. Kubota, H. Kunieda, P. Laurent, F. Lebrun, O. Limousin, M. Loewenstein, K. Long, G. Madejski, Y. Maeda, K. Makishima, M. Markevitch, H. Matsumoto, K. Matsushita, D. McCammon, J. Miller, S. Mineshige, K. Minesugi, T. Miyazawa, T. Mizuno, K. Mori, H. Mori, K. Mukai, H. Murakami, T. Murakami, R. Mushotzky, Y. Nakagawa, T. Nakagawa, H. Nakajima, T. Nakamori, K. Nakazawa, Y. Namba, M. Nomachi, S. O. Dell, H. Ogawa, M. Ogawa, K. Ogi, T. Ohashi, M. Ohno, M. Ohta, T. Okajima, N. Ota, M. Ozaki, F. Paerels, S. Paltani, A. Parmer, R. Petre, M. Pohl, S. Porter, B. Ramsey, C. Reynolds, S. Sakai, R. Sambruna, G. Sato, Y. Sato, P. Serlemitsos, M. Shida, T. Shimada, K. Shinozaki, P. Shirron, R. Smith, G. Sneiderman, Y. Soong, L. Stawarz, H. Sugita, A. Szymkowiak, H. Tajima, H. Takahashi, Y. Takei, T. Tamagawa, T. Tamura, K. Tamura, T. Tanaka, Y. Tanaka, Y. Tanaka, M. Tashiro, Y. Tawara, Y. Terada, Y. Terashima, F. Tombesi, H. Tomida, M. Tozuka, Y. Tsuboi, M. Tsujimoto, H. Tsunemi, T. Tsuru, H. Uchida, Y. Uchiyama, H. Uchiyama, Y. Ueda, S. Uno, M. Urry, S. Watanabe, N. White, T. Yamada, H. Yamaguchi, K. Yamaoka, N. Yamasaki, M. Yamauchi, S. Yamauchi, Y. Yatsu, D. Yonetoku, and A. Yoshida, "The ASTRO-H Mission," in Space Telescopes and Instrumentation 2010: Ultraviolet to Gamma Ray, ser. Proc. SPIE, M. Arnaud, S. S. Murray, and T. Takahashi, Eds., vol. 7732, 2010, p. $77320 Z$.

[14] M. Kokubun, K. Nakazawa, T. Enoto, Y. Fukazawa, K. Gilmore, J. Kataoka, M. Kawaharada, P. Laurent, F. Lebrun, O. Limousin, K. Makishima, T. Mizuno, K. Mori, T. Nakamori, M. Ohno, M. Ohta, G. Sato, H. Tajima, H. Takahashi, T. Takahashi, T. Tanaka, Y. Terada, H. Uchiyama, Y. Uchiyama, S. Watanabe, Y. Yatsu, , and K. Yamaoka, "Hard X-ray imager (HXI) for the ASTRO-H Mission," in Space Telescopes and Instrumentation 2010: Ultraviolet to Gamma Ray, ser. Proc. SPIE, M. Arnaud, S. S. Murray, and T. Takahashi, Eds., vol. 7732, 2010, p. 773215.

[15] H. Tajima, T. Nakamoto, T. Tanaka, S. Uno, T. Mitani, E. do Couto e Silvia, Y. Fukazawa, T. K. G. Madejski, D. Marlow, K. Nakazawa, M. Nomachi, Y. Okada, and T. Takahashi, "Performance of a Low Noise Front-end ASIC for Si/CdTe Detectors in Compton Gamma-ray Telescope," IEEE Trans. Nucl. Sci., vol. 51, pp. 842-847, 2004.

[16] S. Watanabe, S. Ishikawa, H. Aono, S. Takeda, H. Odaka, M. Kokubun, T. Takahashi, K. Nakazawa, H. Tajima, M. Onishi, and Y. Kuroda, "High Energy Resolution Hard X-Ray and Gamma-Ray Imagers Using CdTe Diode Devices," IEEE Trans. Nucl. Sci., vol. 56, pp. 777-782, 2009. 